\title{
Prevalence of Cryptosporidium in Children Under 13 Years of Age With Acute Diarrhea in Tabriz, Iran
}

\author{
Yaghoub Firouzivand ${ }^{1}$, Yagoob Garedaghi $i^{2^{\star}}$, Hamidreza Hassanzadeh Khanmiri ${ }^{\circledR}$ \\ 1Department of Pathobiology, Malekan Branch, Islamic Azad University, Malekan, Iran \\ 2Department of Parasitology, Tabriz Branch, Islamic Azad University, Tabriz, Iran \\ ${ }^{3}$ Department of Basic Sciences, Maragheh Branch, Islamic Azad University, Maragheh, Iran
}

\begin{abstract}
Introduction: Cryptosporidium is a coccidian parasite that causes diarrhea in animals and humans, especially children. Although several studies have been done on Cryptosporidium infection in some parts of Iran, this parasite is not examined in routine parasitological tests. Therefore, the aim of this study was to determine the frequency of Cryptosporidium infection in children with diarrhea in Tabriz, Iran.

Materials and Methods: In this study, 100 fecal samples of children under 13 years of age with acute diarrhea who referred to medical diagnostic laboratories in Tabriz during 2018 were examined for Cryptosporidium infection. The samples were first concentrated by formalin-ether method and after staining with modified acid fast method, they were examined by light microscope.

Results: In this study, 3 children (3\%), 2 boys, 3 years old and 5 years old, and a 3-year-old girl had cryptosporidiosis.

Conclusion: The results of the present study show the presence of Cryptosporidium infection in children in Tabriz. The very low percentage of infection with this parasite indicates that this parasite is not yet considered as a health problem in this area.

Keywords: Cryptosporidium, Children, Acute diarrhea, Tabriz
\end{abstract}

Received: September 12, 2020, Accepted: December 20, 2020, ePublished: January 1, 2021

\section{Introduction}

Cryptosporidiosis is a parasitic disease caused by a protozoan parasite of the genus Cryptosporidium. Cryptosporidium parasites in the form of oocytes with a diameter of about $5 \mu \mathrm{m}$, including four sporozoites, were found in the environment or feces of infected people. This parasite is one of the important causes of diarrhea in humans, especially in children and people with dysfunctional immune system, as well as in animals (1). In the early 1970s, veterinarians realized the importance of this protozoan in causing acute diarrhea in calves. The disease can range from moderate to severe in terms of the severity of symptoms, the site of infection in the body, nutritional status, and immune system. If left untreated, it can lead to death. Of the 20 known species of this parasite, at least eight species, including C. hominis, C. parvum, C. meleagridis, C. felis, C. canis, C. suis, C. muris, and C. andersoni, cause disease in humans. C. parvum and $C$. hominis are the most important species causing disease in humans (2). In immunocompromised individuals, this protozoan is more common at the end of the small intestine and the beginning of the colon, which causes destruction of the host's intestinal cells. Giardia and Cryptosporidium are two common parasites that cause diarrhea in humans, with a prevalence of 1 to $3 \%$ in developed countries and $4 \%$ to $17 \%$ in developing countries. In a recent study conducted in southern India, Cryptosporidium has been reported as one of the most common causes of diarrhea in children (3). The onset of this disease in early childhood may be associated with developmental and physical disorders as well as cognitive disorders. If infected, children may develop severe diarrhea due to a weakened immune system, which is usually self-limiting. At the same time, due to the health status and other factors affecting the child's safety, treatment may be needed in a group of them. Numerous epidemics of this disease have also made this parasite and the resulting disease more important (4). The parasite is mainly transmitted through feces and mouth; however, transmission through airborne particles has also been reported (5).

Considering the life cycle of Cryptosporidium, its transmission from livestock to humans as well as humans 
to humans through contact with the contaminated animals and human feces (contaminated water and food), and the importance of this intestinal protozoan in recent years, the identification and treatment of cryptosporidiosis, especially in children and patients with diarrhea, may play an effective role in maintaining public health $(6,7)$. The prevalence of cryptosporidiosis is higher in patients with diarrhea than in the whole community and Cryptosporidium is the third or fourth known cause of diarrhea in humans $(8,9)$, which causes long-term diarrhea in children and reduced growth and weight. Due to the lack of identification of an effective treatment method to deal with it, its treatment is symptomatic (10).

Due to the lack of studies in recent years on the status of this protozoan in Tabriz, the present study was conducted to determine the prevalence of Cryptosporidium infection in children with acute diarrhea referred to medical diagnostic laboratories in Tabriz.

\section{Materials and Methods}

In this descriptive study, the sample size of children with diarrhea was calculated to be 100 . Freshly collected fecal samples of 100 children under 13 years of age who referred to medical diagnostic laboratories in Tabriz due to acute diarrhea from May 1 to the end of March 2018 were collected by convenience sampling method and taken to the laboratory in less than one hour. Stool samples were collected from Tabriz and 6 villages around Tabriz. While sampling, information about age, gender, breastfeeding status, drinking water status, contact with livestock and, place of residence (urban, rural) were collected from their parents. Children who used antiparasitic drugs or were treated with antibiotics were excluded from the study. All samples were examined by formalin-ether method. In this method, $1 \mathrm{~g}$ of fecal sample was poured into $10 \mathrm{~mL}$ of $10 \%$ formalin and passed through two layers of surgical gauze into the centrifuge tube. Then, 3 $\mathrm{mL}$ of diethyl ether was added to each tube and stirred vigorously to obtain a uniform mixture. The resulting mixture was then centrifuged and the supernatant was discarded, while the sediment was placed on a glass slide and dried in air. Modified fast acid staining method was used to stain the slides. The alkaline fuchsin solution was poured onto the slides and gently heated using an alcohol lamp without boiling. The slides were then incubated at room temperature for 5 minutes, washed with plain water, and stained with $1 \%$ sulfuric acid solution for 1 to 2 minutes. The slides were finally rinsed once more with water at room temperature. They were then stained with methylene blue for 1 minute and washed again with water. The slides were air-dried and examined for the presence of Cryptosporidium using immersion oil and $\times 1000$ magnification of a light microscope (11).

Figure 1 shows Cryptosporidium oocysts (4 to $6 \mu \mathrm{m}$ ) in bright red on a blue background.

\section{Results}

In this study, out of 100 children studied, 58 were boys and 42 were girls (42). The mean age of children was 6.2 years. Cryptosporidium infection was observed in 3 cases (3\%), i.e., 2 boys ( 3 years old and 5 years old) and 1 girl ( 3 years old), all of whom were under 6 years of age. A total of 78 children were urban residents (78\%) and the rest were rural residents $(22 \%)$. Two of the observed infection cases belonged to children living in urban areas and one case belonged to children living in rural areas. A total of 19 people had contact with livestock, one of whom was infected with the parasite. Additionally, 79 people had access to urban water supply, of which 3 children were infected with the parasite, and 21 people had access to rural water supply. A total of 96 children were breastfed, a 3-year-old boy infected with Cryptosporidium. A 3-yearold girl infected with the parasite has also received breast milk during infancy.

\section{Discussion}

The results of this study show the presence of Cryptosporidium infection in children in Tabriz. Due to the acceptance of the modified acid fast method as the gold standard method for the detection of Cryptosporidium oocytes, this method has been used in this study.

Out of 100 children with gastroenteritis studied by fast acid staining method, 3\% showed Cryptosporidium infection, which is very close to the reported percentage of children with diarrhea in Tehran (2.4\%).

In a study conducted on children with gastroenteritis in Qazvin, the prevalence of infection was reported to be lower $(0.3 \%)$ compared to this study (12).

Different rates of infection in children with diarrhea have been reported from different parts of Iran, ranging from 3 to $7 \%$. The difference in the prevalence of this parasite can depend on the level of public health awareness, lifestyle, cultural and economic level of the community, the environment (urban or rural), and

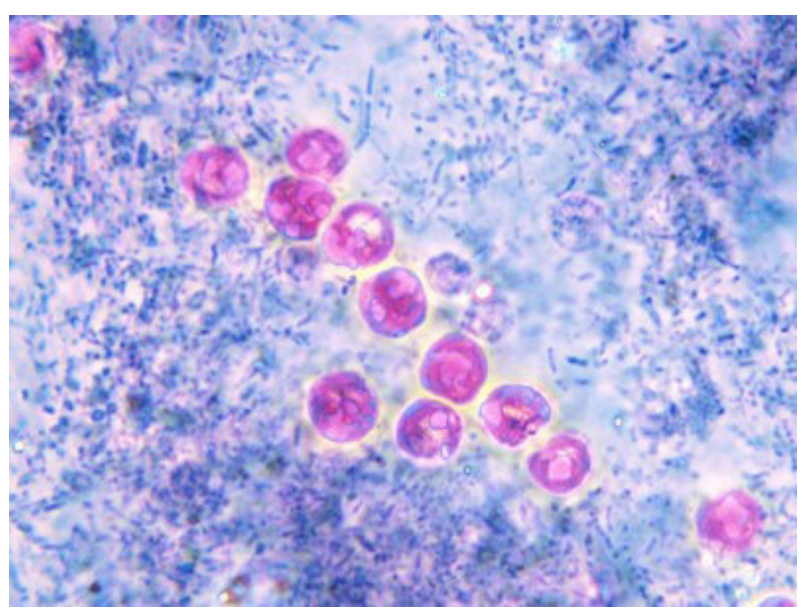

Figure 1. Modified Acid-fast Staining. Note: Cryptosporidium image of patient specimen with a magnification of $\times 1000$. 
geographical climate $(13,14)$.

In the present study, the prevalence of infection with this protozoan in male $(1.97 \%)$ and female $(1.04 \%)$ children is different, which is different from studies conducted in Iran and abroad, which have a similar chance of infection. However, it is similar to the results of studies conducted in Korea and Slovenia, which have shown a higher prevalence among boys (15).

In a study on 700 children under the age of 15 referred to the studied hospitals in Kermanshah, 15 cases of Cryptosporidium infection were observed (16).

In addition, in a study conducted by Dabirzadeh et al in Zahedan, the rate of infection was higher in boys than in girls, and this difference was not statistically significant, which is consistent with the present study (17).

In a study conducted by Gatei et al in Kenya on children under 5 years of age, the highest rate of infection was reported in children aged 1 to 2 years (18).

Moreover, in a study conducted in India, the prevalence of Cryptosporidium parasite in children with diarrhea varied between 1 and 19\%.

In a study conducted by Saneian et al on children under 10 years of age in Isfahan, it was found that there is no significant relationship between the prevalence and age, which is similar to the present study (19).

In a study conducted by Maleki et al in Khorramabad, the highest rate of infection was found among children aged 6 months to 1 year and the lowest among children aged 2 to 10 years (20).

According to the above-mentioned studies, it seems that due to the improvement of the immune system in children with age, the possibility of infection with this parasite decreases.

In this study, there was no significant difference between urban and rural children, which is similar to the findings of studies conducted by Gholami et al in Mazandaran (21), Javidmehr et al (22) and Ranjbar-Bahadori et al in Babol (23).

In the present study, infection was observed in $7.34 \%$ of people who had contact with livestock. Additionally, in a study conducted by Al-Shamiri et al in Yemen, a high prevalence of the parasite was observed in people who had contact with livestock (24).

Due to the very low percentage of infection observed in this study, it seems that the relationship between the infection rate and other variables affecting the mentioned clinical disease needs to be examined with a larger sample size.

\section{Conclusion}

The results of this study, which was conducted in Tabriz, showed the presence of Cryptosporidium infection in this city. Therefore, given the zoonotic nature of this protozoan and its transmission from livestock to humans and considering its threats to the health of individuals and society, children should be seriously avoided from contact with livestock. The very low percentage of infection with this parasite in children with diarrhea in Tabriz indicates that this parasite is not yet considered as a health problem in this area, which may be due to the increase in public health awareness, improvement and promotion of lifestyle, and increase in cultural and economic levels in urban and rural communities of Tabriz in recent years.

\section{Conflict of Interests}

The authors declare that they have no conflict of interests.

\section{Ethical Issues}

In this research, ethical considerations have been fully observed.

\section{Acknowledgements}

We would like to express our deep gratitude to Islamic Azad University, Tabriz Branch, for offering valuable theoretical and practical contributions to the research team in the present study.

\section{Authors' Contributions}

YG did the writing and editing of the manuscript. YF and HHK did data collection and statistical analysis.

\section{Funding}

The authors received no financial support for the research, authorship, and publication of this article.

\section{References}

1. Fayer R, Morgan U, Upton SJ. Epidemiology of Cryptosporidium: transmission, detection and identification. Int J Parasitol. 2000;30(12-13):1305-22. doi: 10.1016/s0020-7519(00)00135-1

2. Cama VA, Bern C, Roberts J, et al. Cryptosporidium species and subtypes and clinical manifestations in children, Peru. Emerg Infect Dis. 2008;14(10):1567-74. doi: 10.3201/ eid1410.071273

3. Mondal D, Haque R, Sack RB, Kirkpatrick BD, Petri WA Jr. Attribution of malnutrition to cause-specific diarrheal illness: evidence from a prospective study of preschool children in Mirpur, Dhaka, Bangladesh. Am J Trop Med Hyg. 2009;80(5):824-6.

4. WHO. WHO child growth standards: methods and development. 2006. http://www.who.int/childgrowth/ standards/technical_report/en/. Accessed July 27, 2017.

5. Adams RB, Guerrant RL, Zu S, Fang G, Roche JK. Cryptosporidium parvum infection of intestinal epithelium: morphologic and functional studies in an in vitro model. J Infect Dis. 1994;169(1):170-7. doi: 10.1093/infdis/169.1.170

6. Mead JR. Challenges and prospects for a Cryptosporidium vaccine. Future Microbiol. 2010;5(3):335-7. doi: 10.2217/ fmb.09.115

7. Liu J, Platts-Mills JA, Juma J, et al. Use of quantitative molecular diagnostic methods to identify causes of diarrhoea in children: a reanalysis of the GEMS casecontrol study. Lancet. 2016;388(10051):1291-301. doi: 10.1016/s0140-6736(16)31529-x

8. Liu J, Kabir F, Manneh J, et al. Development and assessment of molecular diagnostic tests for 15 enteropathogens 
causing childhood diarrhoea: a multicentre study. Lancet Infect Dis. 2014;14(8):716-24. doi: 10.1016/s14733099(14)70808-4

9. Efstratiou A, Ongerth JE, Karanis P. Waterborne transmission of protozoan parasites: review of worldwide outbreaks - an update 2011-2016. Water Res. 2017;114:1422. doi: 10.1016/j.watres.2017.01.036

10. Squire SA, Ryan U. Cryptosporidium and Giardia in Africa: current and future challenges. Parasit Vectors. 2017;10(1):195. doi: 10.1186/s13071-017-2111-y

11. Mor SM, Tzipori S. Cryptosporidiosis in children in subSaharan Africa: a lingering challenge. Clin Infect Dis. 2008;47(7):915-21. doi: 10.1086/591539

12. Shirley DA, Moonah SN, Kotloff KL. Burden of disease from cryptosporidiosis. Curr Opin Infect Dis. 2012;25(5):55563. doi: 10.1097/QCO.0b013e328357e569

13. Agholi M, Hatam GR, Motazedian MH. HIV/AIDSassociated opportunistic protozoal diarrhea. AIDS Res Hum Retroviruses. 2013;29(1):35-41. doi: 10.1089/ aid.2012.0119

14. Striepen B. Parasitic infections: time to tackle cryptosporidiosis. Nature. 2013;503(7475):189-91. doi: $10.1038 / 503189$ a

15. Vouking MZ, Enoka P, Tamo CV, Tadenfok CN. Prevalence of intestinal parasites among HIV patients at the Yaoundé Central Hospital, Cameroon. Pan Afr Med J. 2014;18:136. doi: 10.11604/pamj.2014.18.136.3052

16. Medema GJ, Schijven JF. Modelling the sewage discharge and dispersion of Cryptosporidium and Giardia in surface water. Water Res. 2001;35(18):4307-16. doi: 10.1016/s00431354(01)00161-0

17. Dabirzadeh M, Baghaei M, Bokaeyan M, Goodarzei MR. Study of Cryptosporidium in children below five years of age with diarrhea in referring Ali-Asghar Pediatric Hospital of Zahedan. J Gorgan Univ Med Sci. 2003;5(1):549. [Persian].

18. Gatei W, Wamae CN, Mbae C, et al. Cryptosporidiosis: prevalence, genotype analysis, and symptoms associated with infections in children in Kenya. Am J Trop Med Hyg. 2006;75(1):78-82.

19. Saneian H, Yaghini O, Yaghini A, Modarresi MR, Soroshnia M. Infection rate of Cryptosporidium parvum among diarrheic children in Isfahan. Iran J Pediatr. 2010;20(3):343-7.

20. Maleki S, Nayebzadeh H, Shafizadeh F. A survey on prevalence rate of cryptosporidiosis among children with diarrhea in Khorram-Abad. Tehran Univ Med J. 2005;63(2):151-9. [Persian].

21. Gholami S, Hamzeh Ali AA, Khalilian A, et al. Frequency of cryptosporidiosis among gastroenteritic patients. J Mazandaran Univ Med Sci. 2012;21(Suppl 1):261-70. [Persian].

22. Javidmehr A, Garedaghi Y, Sioufi AB. Assessment of Cryptosporidium in patients with gastroenteritis by modified Ziehl-Neelsen staining method in East Azerbaijan province of Iran during 2018-2019. Int J Med Parasitol Epidemiol Sci. 2020;1(3):56-60. doi: 10.34172/ ijmpes.2020.17

23. Ranjbar-Bahadori S, Sangsefidi H, Shemshadi B, Kashefinejad M. Cryptosporidiosis and its potential risk factors in children and calves in Babol, north of Iran. Trop Biomed. 2011;28(1):125-31.

24. Al-Shamiri A, Al-Zubairy A, Al-Mamari R. The prevalence of Cryptosporidium spp. in children, Taiz District, Yemen. Iran J Parasitol. 2010;5(2):26-32.

(C) 2021 The Author(s); This is an open-access article distributed under the terms of the Creative Commons Attribution License (http:// creativecommons.org/licenses/by/4.0), which permits unrestricted use, distribution, and reproduction in any medium, provided the original work is properly cited. 